\title{
ON THE CLASSIFICATION OF CRYSTALS.
}

BY PROFESSOR PAUL SAUREL.

(Read before the American Mathematical Society, December 29, 1910.)

THE determination of the various types of crystal symmetry is a problem which has attracted the attention of many physicists and mathematicians. The first solutions of the problem are those of Hessel,* of Bravais, $\nmid$ and of Gadolin, $\$$ while the latest and most elegant is that of Lorentz. § In what follows I should like to show that by combining theorems due to Curie and to Lorentz it is very easy to enumerate and to remember the different types of symmetry.

A few preliminaries are necessary. In the first place, if the line joining two points $P$ and $P^{\prime}$ is bisected by a point $O$, we shall say that $P^{\prime}$ is the inverse of $P$ with respect to $O$. In the next place we shall use the phrase inversion with respect to a point to denote the operation which consists in replacing every point of a figure by its inverse with respect to the given point.

Let us consider any system of points. If the system is such that rotation through a suitable angle about a line $O A$ transforms the system of points into itself, we shall say that $O A$ is an axis of direct symmetry. It is obvious that if rotation through an angle $\alpha$ transforms the system into itself, rotation through any multiple of $\alpha$ will al so transform the system into itself. It follows that the number of different rotations which transform the system into itself will be infinite unless $\alpha$ is commensurable with $2 \pi$. Accordingly, if we limit ourselves to systems which admit of a finite number only of transformations, $\alpha$ must be equal to $2 \pi m / q$, where $m$ and $q$ are integers prime to each other.

* J . F. C. Hessel, Krystallometrie oder Krystallonomie und Krystallographie; Gehler's Physikalisches Wörterbuch, vol. 5, 1830, p. 1062; Ostwald's Klassiker der exakten Wissenschaften, no. 88.

† A. Bravais, "Mémoire sur les polyèdres de forme symétrique," Journal de mathématiques pures et appliquées, vol. 14 (1849), p. 141; Ostwald's Klassiker der exakten Wissenschaften, no. 17.

¥A. Gadolin, "Mémoire sur la déduction d’un seul principe de tous les systèmes crystallographiques," Acta Societatis Scientiarum Fennicae, Helsingfors, vol. 9 (1871), p. 1; Ostwald's Klassiker der exakten Wissenschaften, no. 75.

\& H. A. Lorentz, "Über die Symmetrie der Kristalle", Abhandlungen über theoretische Physik, vol. 1 (1907), p. 299. 
Morever, it can be shown without difficulty that the different rotations about $O A$ correspond to the first $q-1$ multiples of the angle $2 \pi / q$. The axis $O A$ is said to be of order $q$, and the rotations about it may be performed in either direction. It is clear that if $O A$ is an axis of direct symmetry of order $q$, the same is true of $O A^{\prime}$, the inverse of $O A$ with respect to $O$.

The system of points may be such that it is possible to draw through a point $O$ two sets of rectangular coordinate axes, one right-handed and the other left-handed, in such a way that to every point of the system whose coordinates with respect to one set of axes are the numbers $x, y, z$, there corresponds a point of the system whose coordinates with respect to the other set of axes are the same numbers $x, y, z$. When this is the case, it is easy to see that it is possible to transform the system into itself by means of a rotation followed by an inversion. Indeed, it is clear that a rotation can be found which will bring one set of coordinate axes into coincidence with the inverses of the other set; this rotation, followed by an inversion with respect to $O$, will obviously transform every point of the system into a point of the system. If the rotation is unnecessary, so that a mere inversion with respect to $O$ transforms the system into itself, we shall say that $O$ is a center of symmetry. In the general case, where a rotation is necessary, we shall call the axis of rotation an axis of inverse symmetry.

If, as before, we limit ourselves to systems which admit of a finite number only of transformations, it follows that the angle of rotation associated with an axis of inverse symmetry must be equal to $2 \pi m / q$, where $m$ and $q$ are integers prime to each other. If $q$ is odd, it can be shown that the axis of inverse symmetry is equivalent to an axis of direct symmetry of order $q$ and a center of symmetry ; if, on the contrary, $q$ is even, the various operations connected with the axis of inverse symmetry are equivalent to the repetition of a rotation through an angle $2 \pi / q$ accompanied by an inversion.

To establish this, we observe that the rotation about the axis of inverse symmetry may precede or follow the inversion; the result in each case is the same. Moreover, two successive inversions always transform a system into itself. Accordingly, $q$ rotations through an angle $2 \pi m / q$, accompanied by $q$ inversions, are equivalent to rotation through an angle $2 \pi \mathrm{m}$ followed by $q$ inversions ; if $q$ is odd, the operation is thus equivalent to a single inversion and the system possesses a center of sym- 
metry. From this it follows immediately that a mere rotation through the angle $2 \pi m / q$ transforms the system into itself. Accordingly, an axis of inverse symmetry of odd order is equivalent to an axis of direct symmetry of the same order and a center of symmetry.

Again, the repetition $k$ times of the operation of rotation through an angle $2 \pi \mathrm{m} / q$ and inversion is equivalent to a single rotation of angle $2 \pi m k / q-2 \pi l$, where $l$ is an integer, followed by $k$ inversions. Since

$$
\frac{2 \pi m k}{q}-2 \pi l=\frac{2 \pi}{q}(m k-q l),
$$

and since we can always choose $k$ and $l$ so that

$$
m k-q l= \pm 1
$$

it follows that one of the operations connected with the axis of inverse symmetry consists in a rotation of angle $2 \pi / q$ followed by $k$ inversions. From the last equation it follows that $k$ must be prime to $q$; accordingly, when $q$ is even, $k$ must be odd. Thus, when $q$ is even, all the operations connected with the axis of inverse symmetry are repetitions of a rotation through an angle $2 \pi / q$ accompanied by an inversion. There are $q-1$ different operations and no one of them is an inversion.

It is now easy to see that the crystallographic problem of the determination of the various possible types of crystal symmetry is equivalent to the problem of determining the various sets of axes of direct and of inverse symmetry passing through a common point, which a system can possess ; the point of intersection of the axes may or may not be a center of symmetry.

For this purpose it is sufficient to recall that crystals possess two kinds of axes of symmetry. An axis of symmetry of the first kind is characterized by the fact that the physical properties of the crystal with respect to any fixed directions in space remain unchanged when the crystal is turned through a suitable angle about the axis. Accordingly, if we consider a portion of the crystal bounded by a spherical surface and if we draw the axis of symmetry through the center of this sphere, we may say that a rotation about the axis transforms the crystal into itself.

An axis of symmetry of the second kind is characterized by the fact that if the crystal is turned through a suitable angle about such an axis, its physical properties in its new position 
and with respect to any fixed directions in space are identical with the properties of the crystal in its first position and with respect to the fixed directions which are the inverse of those just mentioned. Accordingly, if, as before, we consider a portion of the crystal bounded by a spherical surface, and if, through the center, we draw an axis of symmetry of the second kind, we may say that a rotation about this axis, followed by an inversion with respect to the center of the sphere, transforms the crystal into itself.

We shall now proceed to the enumeration of the different sets of axes of direct and of inverse symmetry, passing through a common point, that a system can possess. From what precedes, it is obvious that this problem is identical with the problem of finding all the finite groups of operations that can be generated by rotations about a point and inversion with respect to it.

Let us begin by finding the groups that consist of rotations only; it will then be easy to find the groups that contain inversions also. In the first place, it is clear that, if $O A$ be an axis of direct symmetry of order $q$, the different positions which $O A$ takes when the system is subjected to all the rotations of the group are also axes of direct symmetry of order $q$. Every axis thus belongs to a definite set of equivalent axes and the various axes of the system can be grouped into one or more sets of equivalent axes. In enumerating the axes of direct symmetry we must distinguish between $O A$ and its inverse $O A^{\prime} ; O A$ and $O A^{\prime}$ will belong to the same or to different sets of equivalent axes according as it is or is not possible to bring $O A$ into coincidence with $O A^{\prime}$.

We can now establish the following two theorems which are due to Curie* and which suffice to determine the possible groups of rotations.

THEOREM I. If $n$ be the number of different rotations, including the identical rotation, which transform a system of points into itself, and if $p$ be the number of different positions which an axis of order $q$ assumes when the system is subjected to these $n$ rotations, then

$$
p q=n \text {. }
$$

* P. Curie, "Sur les questions d'ordre," Bulletin de la Société minéralogique de France, vol. 8 (1884), p. 89 ; Oeuvres, p. 70. "Sur les répétitions et la symétrie," Comptes rendus de l'Académie des Sciєnces, vol. 100 (1885), p. 393 ; Oeuvres, p. 114. 
THEOREm II. If a system possesses a set of $p$ equivalent axes of order $q, a$ set of $p^{\prime}$ equivalent axes of order $q^{\prime}, a$ set of $p^{\prime \prime}$ equivalent axes of order $q^{\prime \prime}, \ldots$, and if $k$ be the number of these sets, then

$$
p(q-1)+p^{\prime}\left(q^{\prime}-1\right)+p^{\prime \prime}\left(q^{\prime \prime}-1\right)+\cdots=2(n-1),
$$
or finally

$$
p+p^{\prime}+p^{\prime \prime}+\cdots=(k-2) n+2,
$$

$$
\frac{1}{q}+\frac{1}{q^{\prime}}+\frac{1}{q^{\prime \prime}}+\cdots=k-2+\frac{2}{n} .
$$

To establish equation (1) it is sufficient to observe that for each of the $p$ positions which the axis of rotation under consideration can assume, the system can assume any one of $q$ positions obtained by rotation about this axis; the system can thus be transformed into itself by any one of $p q$ different operations. Moreover, it is obvious that the $p q$ different positions thus obtained are the only ones which the system can take; thus $p q$ is equal to $n$.

To establish equation ( 2 ) we observe that the system can be transformed into itself by any one of $q-1$ rotations about each of the $p$ axes of the first set, by any one of $q^{\prime}-1$ rotations about each of the $p^{\prime}$ axes of the second set, by any one of $q^{\prime \prime}-1$ rotations about each of the $p^{\prime \prime}$ axes of the third set, $\cdots$, and that in this enumeration every rotation, except the identical rotation, has been counted twice; $O A$ and its inverse $O A^{\prime}$ may belong to the same or to different sets of axes, in either case the rotations about $A A^{\prime}$ have been counted twice. Equation (3) follows without difficulty from equations (1) and (2,) and in like manner equation (4) follows from equations (1) and (3).

The solutions of equations (1) and (4) will give us all the sets of axes of direct symmetry that can coexist. There is no difficulty in finding these solutions; it is found that when $k=1$ or $k>3$ there are no solutions, and the results for $k=2$ and $k=3$ are given in the following table : 


\begin{tabular}{|c|c|c|c|c|c|c|c|}
\hline$k$ & $p$ & $p^{\prime}$ & $p^{\prime \prime}$ & $q$ & $q^{\prime}$ & $q^{\prime \prime}$ & $n$ \\
\hline 2 & 1 & 1 & & $n$ & $n$ & & $n$ \\
\hline & $m$ & $m$ & 2 & 2 & 2 & $m$ & $2 m$ \\
\hline 3 & 6 & 4 & 4 & 2 & 3 & 3 & 12 \\
\hline & 12 & 8 & 6 & 2 & 3 & 4 & 24 \\
\hline & 30 & 20 & 12 & 2 & 3 & 5 & 60 \\
\hline
\end{tabular}

We shall now show that corresponding to each solution in the above table there exists one and but one arrangement of the axes of symmetry in space. When $k=2$, there are two sets of equivalent axes each consisting of a single axis of order $n$; it is obvious that these two axes must be inverses of each other. The geometric configuration corresponding to this solution thus exists and it is unique. The corresponding groups of rotations are called the cyclic groups.

When $k=3$, let us draw a unit sphere about $O$ as a center and let us mark on the sphere the points where the three sets of axes of symmetry pierce it. It is well known that if we construct any spherical triangle $A B C$, a rotation about $O A$ through an angle $2 A$, followed by a rotation about $O B$ through an angle $2 B$, is equivalent to a rotation about $O C$ through an angle $2 C$. Accordingly, if $O A$ and $O B$ are axes of symmetry of orders $q$ and $q^{\prime}$ and if the angles $A$ and $B$ are respectively equal to $\pi / q$ and $\pi / q^{\prime}$, the line $O C$ will also be an axis of symmetry of the system and the half-angle of rotation associated with it will be equal to $C$ or to some aliquot part of $C$. We shall establish the following theorem:

THeOREM III. If $O A$ and $O B$ are axes of symmetry of orders $q$ and $q^{\prime}$ belonging to different sets of equivalent axes and if the angle between $O A$ and $O B$ is not greater than the angle between any two axes of symmetry belonging to different sets, then, if we construct the spherical triangle $A B C$ with angles $A$ and $B$ respectively equal to $\pi / q$ and $\pi / q^{\prime}$, the line $O C$ will be an axis of symmetry of order $q^{\prime \prime}$ belonging to the third set of equivalent axes and the angle $C$ will be equal to $\pi / q^{\prime \prime}$.

In the first place, the half-angle of rotation associated with the axis $O C$ is equal to the angle $C$ and not to an aliquot part 
of $C$. For if an aliquot part of $C$ were the half-angle of rotation associated with the axis $O C$, we could draw through $C$ an arc making this angle with $A C$ and thus form a triangle $A C D$; the line $O D$, lying between $O A$ and $O B$, would thus, contrary to hypothesis, be an axis of symmetry.

In the next place, it is obvious that if the angle $C$ differs from the angles $A$ and $B$, the axis of symmetry $O C$ belongs to the third set of equivalent axes. It remains to prove that this is the case even when the angle $C$ is equal to one of the angles $A$ or $B$. Accordingly, let us suppose that in the triangle $A B C$ the angles $B$ and $\dot{C}$ are equal and that $O B$ and $O C$ belong to the same set of equivalent axes. It is easy to see that no axis belonging to the third set of axes can pierce the sphere within the triangle $A B C$ or on either of the sides $A B$ and $A C$, for any such axis would make with $O A$ a smaller angle than does $O B$. This becomes evident if we remember that no one of the angles of the triangle is greater than $\frac{1}{2} \pi$ and that consequently the perpendiculars from the vertices to the opposite sides do not fall without the triangle. Further, no axis of the third set can meet the side $B C$. The reason just given applies here also except when $A B$ and $A C$ are quadrants; but in that case the arc $B C$ cannot be greater than a quadrant since the angle $A$ is not greater than $\frac{1}{2} \pi$, and consequently any axis meeting the side $B C$ would form with $O B$ an angle less than the angle between $O B$ and $O A$. Thus, no axis belonging to the third set can pierce the sphere within the triangle $A B C$ or on its perimeter.

Let us now construct a triangle $B C A_{1}$ equal to $B C A$ and having the side $B C$ in common. The vertex $A_{1}$ corresponds to an axis belonging to the same set as $O A$, for $O A_{1}$ is the position which $O A$ takes when it is revolved about $O B$ through an angle $2 B$. In like manner, we can construct triangles $A C B_{1}$ and $A B C_{1}$ equal to $A B C$; the vertices $B_{1}$ and $C_{1}$ will correspond to axes belonging to the same set as $O B$ and $O C$. By repeating this construction we obtain a network of triangles the vertices of which correspond to axes belonging to the same sets as $O A, O B, O C$. This network must cover the sphere completely and there can be no overlapping. Indeed, it is obvious that the construction can be continued as long as any portion of the surface is uncovered. On the other hand there can be no overlapping, for, if there were, there would lie within a triangle such as $A B C$ a point $D$ corresponding to an axis belonging to 
one of the first two sets. If we remember that the angles $A$, $B, C$ are not greater than $\frac{1}{2} \pi$ and that consequently the perpendiculars from the vertices to the opposite sides do not fall without the triangle, it is clear that $D A$ and $D B$ are each less than $A B$. We should then have, contrary to the hypothesis, an axis nearer to $O A$ or to $O B$ than these two are to each other.

The sphere has thus been covered by a network of triangles equal to $A B C$, the vertices of which correspond to axes of the first and second sets. Moreover, it has been shown that no axis of the third set can pierce the triangle $A B C$. In like manner it can be shown that no axis of the third set can pierce a triangle belonging to the network of equal triangles. There is thus no place for the axes belonging to the third set. We must therefore abandon the hypothesis that $O C$ is an axis belonging to the second set and our Theorem III is established.

Let us now consider a triangle $A B C$ of the kind described in Theorem III. By means of the construction described above we can obtain a set of triangles equal to or symmetrical with $A B C$ and the vertices of these triangles will correspond to axes belonging to the three sets of axes. Moreover, it can be shown as above that this network of triangles will cover the sphere completely and that there will be no overlapping. We are thus led to the problem of determining the values of $q, q^{\prime}, q^{\prime \prime}$ such that we can cover the sphere with a set of triangles whose angles are $\pi / q, \pi / q^{\prime}, \pi / q^{\prime \prime}$. Since the area of one of these triangles is

$$
\frac{\pi}{q}+\frac{\pi}{q^{\prime}}+\frac{\pi}{q^{\prime \prime}}-\pi,
$$

we must have, if $N$ denote the number of triangles,

$$
N\left(\frac{\pi}{q}+\frac{\pi}{q^{\prime}}+\frac{\pi}{q^{\prime \prime}}-\pi\right)=4 \pi,
$$

or

$$
\frac{1}{q}+\frac{1}{q^{\prime}}+\frac{1}{q^{\prime \prime}}=1+\frac{4}{N} \text {. }
$$

As this equation differs from equation 4 for $k=3$ only in the substitution of $N$ for $2 n$, its solutions can be at once written down. They are given by the following table 


\begin{tabular}{|c|c|c|c|}
\hline$q$ & $q^{\prime}$ & $q^{\prime \prime}$ & $N$ \\
\hline 2 & 2 & $m$ & $4 m$ \\
\hline 2 & 3 & 3 & 24 \\
\hline 2 & 3 & 4 & 48 \\
\hline 2 & 3 & 5 & 120 \\
\hline
\end{tabular}

It is obvious that if there exists a network of triangles corresponding to any one of these solutions it is unique; for the triangle $A B C$ is determined when its angles are given and the other triangles are determined without ambiguity when $A B C$ is given. Now it is well known that there exists a set of triangles corresponding to each of the above solutions. The $4 \mathrm{~m}$ triangles corresponding to the first solution are obtained by dividing the circumference of a great circle into $2 m$ equal parts and connecting the points of division with the poles of the circle. The radii to the poles of the great circle form a set of 2 equivalent axes of order $m$, while the $2 m$ radii to the points of division of the great circle fall into two sets of $m$ equivalent axes of order 2 . The groups of rotations corresponding to this case are called the dihedral groups.

The configuration corresponding to the second solution in the table is found by inscribing in the sphere a pair of polar tetrahedrons. Each vertex of the first tetrahedron lies within a triangle formed by vertices of the other; by connecting each vertex of the first tetrahedron with the vertices and the midpoints of the sides of the surrounding triangle we find 24 triangles whose angles are $\pi / 2, \pi / 3, \pi / 3$. The vertices of the two tetrahedrons correspond to the two sets of 4 equivalent axes of order 3, while the remaining vertices of the triangles correspond to the 6 axes of order 2 . The corresponding group of rotations is called the tetrahedral group.

The configuration corresponding to the third solution in the table is found by inscribing in the sphere a cube and its polar octahedron. Each vertex of the octahedron lies within a quadrilateral formed by vertices of the cube; by connecting each vertex of the octahedron with the vertices and the mid-points of the sides of the surrounding quadrilateral we find 48 triangles whose angles are $\pi / 2, \pi / 3, \pi / 4$. The vertices of the octahe- 
dron correspond to a set of 6 equivalent axes of order 4 , the vertices of the cube correspond to a set of 8 equivalent axes of order 3 , while the remaining vertices of the triangles correspond to the 12 axes of order 2 . The corresponding group of rotations is called the octahedral group.

Finally, the configuration corresponding to the last solution in the table is found by inscribing in the sphere a regular dodecahedron and its polar icosahedron. Each vertex of the icosahedron lies within a pentagon formed by vertices of the dodecahedron; by connecting each vertex of the icosahedron with the vertices and the mid-points of the surrounding pentagon we find 120 triangles whose angles are $\pi / 2, \pi / 3, \pi / 5$. The vertices of the icosahedron correspond to a set of 12 equivalent axes of order 5, the vertices of the dodecahedron correspond to a set of 20 equivalent axes of order 3, while the remaining vertices of the triangles correspond to the 30 axes of order 2. The corresponding group of rotations is called the icosahedral group.

Thus the only groups of rotations are the identical rotation and the cyclic, the dihedral, the tetrahedral, the octahedral, and the icosahedral groups.

As Lorentz * has shown, we can now obtain without difficulty the groups of operations that contain inversions as well as rotations. In the first place, we may associate an inversion with each of the groups of rotations; the system will then possess in addition to the axes of symmetry a center of symmetry.

In the next place, since an axis of inverse symmetry of odd order is equivalent to an axis of direct symmetry of the same order and a center of symmetry, we may now limit ourselves to the consideration of axes of inverse symmetry of even order. Furthermore, since the inversions and rotations about the axes of inverse symmetry must, like the rotations about the axes of direct symmetry, transform the group of axes of symmetry into itself, it follows that the only admissible configurations for the axes of symmetry are the configurations which we found in studying the groups of rotations. The groups of operations containing operations corresponding to axes of inverse symmetry will therefore be found by replacing one or more sets of axes of direct symmetry by axes of inverse symmetry of the same orders. Finally, if we consider a triangle of the kind

\footnotetext{
* L. c.
} 
described in Theorem III, it is easy to see that if one of the three axes $O A, O B, O C$ be replaced by an axis of inverse symmetry, one other must also be replaced by such an axis while the third will remain an axis of direct symmetry.

With these remarks in mind it is easy to enumerate the groups of operations which contain operations corresponding to axes of inverse symmetry. In the first place, we obtain from every cyclic group of even order a new group by replacing the axis of direct symmetry by an axis of inverse symmetry of the same order. In the next place, we obtain from every dihedral group a new group by replacing the two sets of axes of order 2 by axes of inverse symmetry of order 2. Moreover, we obtain a new group from every dihedral group of order $2 m$, when $m$ is an even number equal to or greater than 4 , by replacing the pair of axes of order $m$ and one set of axes of order 2 by an axis of inverse symmetry of order $m$ and axes of inverse symmetry of order 2. Finally, we obtain a new group from the octahedral group by replacing the axes of orders 2 and 4 by axes of inverse symmetry of the same orders. The tetrahedral and icosahedral groups furnish no new groups.

Our results are summarized in the following table, in which the letters $C, D, T, O, I$ are used to recall the cyclic, the dihedral, the tetrahedral, the octahedral, and the icosahedral groups and the subscripts are used to indicate the orders of the corresponding axes of symmetry. A bar over a subscript indicates that the corresponding axes of direct symmetry have been replaced by axes of inverse symmetry.

TyPes OF SYMMETRY CHARACTERIZED BY THE EXISTENCE OF

\begin{tabular}{|c|c|c|}
\hline $\begin{array}{c}\text { Axes of Direct } \\
\text { Symmetry. }\end{array}$ & $\begin{array}{c}\text { Center of Symmetry } \\
\text { and Axes of Direct } \\
\text { Symmetry. }\end{array}$ & $\begin{array}{c}\text { Axes of Direct and of } \\
\text { Inverse Symmetry }\end{array}$ \\
\hline No axis & No axis & $C_{\overline{2 m}}$ \\
\hline$C_{n}$ & $C_{n}$ & $D_{\overline{2}, \overline{2}, m}$ \\
\hline$D_{2,2, m}$ & $D_{2,2, m}$ & $\kappa \geq 0$ \\
\hline$T_{2,3,3}$ & $T_{2,3,3}$ & $O_{\overline{2}, \overline{3}, \overline{4}}$ \\
\hline$O_{2,3,4}$ & $O_{2,3,4}$ & \\
\hline$I_{2,3,5}$ & $I_{2,3,5}$ & \\
\hline
\end{tabular}


Not all of the types of symmetry enumerated in this table are available as types of crystal symmetry, for the law of rational indices limits the acceptable axes of symmetry to those of the orders $2,3,4,6$. With this limitation the table furnishes the 32 types of crystal symmetry, 11 from each of the first two columns and 10 from the third.

New YoRK.

December 4, 1910.

\title{
HORNER'S METHOD OF APPROXIMATION ANTICIPATED BY RUFFINI.
}

\author{
BY PROFESSOR FLORIAN CAJORI.
}

(Read before the Southwestern Section of the American Mathematical Society, November 26, 1910.)

BEFORE the nineteenth century no simple elementary practical process was known of computing the coefficients of an equation whose roots are less by a given constant than the roots of a given affected numerical equation $f(x)=0$. Such a process was invented in the early part of the last century and is contained in the so-called "Horner's method of approximation."

The history of Horner's method, as developed in England, has been traced in detail by Augustus De Morgan.* $\mathrm{He}$ quotes extensively from original sources and shows that, of English rivals (among whom were Theophilus Holdred, Peter Nicholson, and Henry Atkinson), none except perhaps Nicholson advanced methods of approximation that equalled Horner's, and none was entitled to priority over Horner. It is wellknown that the popularization of Horner's process of approximation in England was due to De Morgan and J. R. Young. Except for the efforts of these men, Horner's paper of 1819 in the Philosophical Transactions might have been lost sight of and forgotten. De Morgan was an enthusiast on Horner's method. He taught it with great zeal ; he made sport of Cambridge tutors who were not familiar with it $\nmid \dagger$ the preparation of his historical tract, alluded to above, was evidently a labor

\footnotetext{
* Companion to the [British] Almanac for 1839, Art. "Notices of the progress of the problem of evolution," pp. 34-52.

$\dagger$ A. De Morgan, A Budget of Paradoxes, London, 1872, pp. 292, 375.
} 